\title{
Mass spectrometry imaging 2.0
}

\author{
Tiffany Porta Siegel ${ }^{1}$ (D) $\cdot$ Shane R. Ellis ${ }^{2,3}$
}

Received: 12 March 2021 / Accepted: 12 March 2021 / Published online: 23 March 2021

(C) Springer-Verlag GmbH Germany, part of Springer Nature 2021

Mass spectrometry Imaging (MSI) is a rapidly maturing analytical technology allowing the spatial distribution of many different molecules to be mapped across complex surfaces such as biological tissue sections. It achieves this in a labelfree manner whilst combining all the advantages of modern mass spectrometry (i.e. sensitivity, specificity, structure elucidation capabilities, etc.) and can nowadays achieve spatial resolutions allowing for cellular and, in some cases, even sub-cellular resolution. Since the emergence of biomolecular MSI in the early late 1980s/early 1990s, the field has experienced significant growth and continued to increase its foothold in the chemical, physical and biological sciences (Fig. 1). Over this time, the scientific community has contributed to numerous developments that improved MSI's analytical capabilities as well as methods to deal with the ever-increasing data demands these bring. This rapid growth across both academia and industry has catalyzed increasing interest from instrument vendors who are continuing to develop increasingly advanced commercial instrumentation and software solutions.

This rapidly increasing MSI performance is now being applied in an increasing number of settings and to samples and molecular classes that previously presented significant challenges. However, users' demands constantly evolve and require more resolution, more sensitivity, and more accessible

Published in the topical collection Mass Spectrometry Imaging 2.0 with guest editors Shane R. Ellis and Tiffany Porta Siegel.

Tiffany Porta Siegel

tiffany.porta@gmail.com

Shane R. Ellis

sellis@uow.edu.au

1 Maastricht MultiModal Molecular Imaging (M4i) institute, Division of Imaging Mass Spectrometry, Maastricht University, 6229 ER Maastricht, The Netherlands

2 Molecular Horizons and School of Chemistry and Molecular Bioscience, University of Wollongong, Wollongong, NSW 2522, Australia

3 Illawarra Health and Medical Research Institute, Wollongong, NSW 2522, Australia molecular classes to meet the demands of new applications. These increasing demands necessitate increasingly sophisticated data analysis routines to allow one to extract all the meaningful data from large datasets that are now routinely many GBs in size.

The 19 articles in this topical collection entitled "Mass Spectrometry Imaging 2.0" encompass many MSI-related techniques and include critical reviews of highly active and growing areas of research as well as communications and research papers describing advances across all areas of MSI, including sample preparation, new experimental approaches, as well as developments in quantitation and data analysis.

We sincerely thank all the authors for their timely contributions and the reviewers for critically assessing and improving these high-quality papers for this special topical collection on "Mass Spectrometry Imaging 2.0" of Analytical and Bioanalytical Chemistry, especially under the challenging circumstances that affected all of us in 2020 . We are also incredibly grateful to the editorial office for the efforts in helping compile this collection. We are very proud of the quality of contributions to this topical collection, and we hope it serves as a valuable resource for both new and experienced researchers and users in the field. 
Fig. 1 Number per year of scientific papers containing topical keywords « Mass Spectrometry Imaging » according to a Web of Science search on February 15, 2021

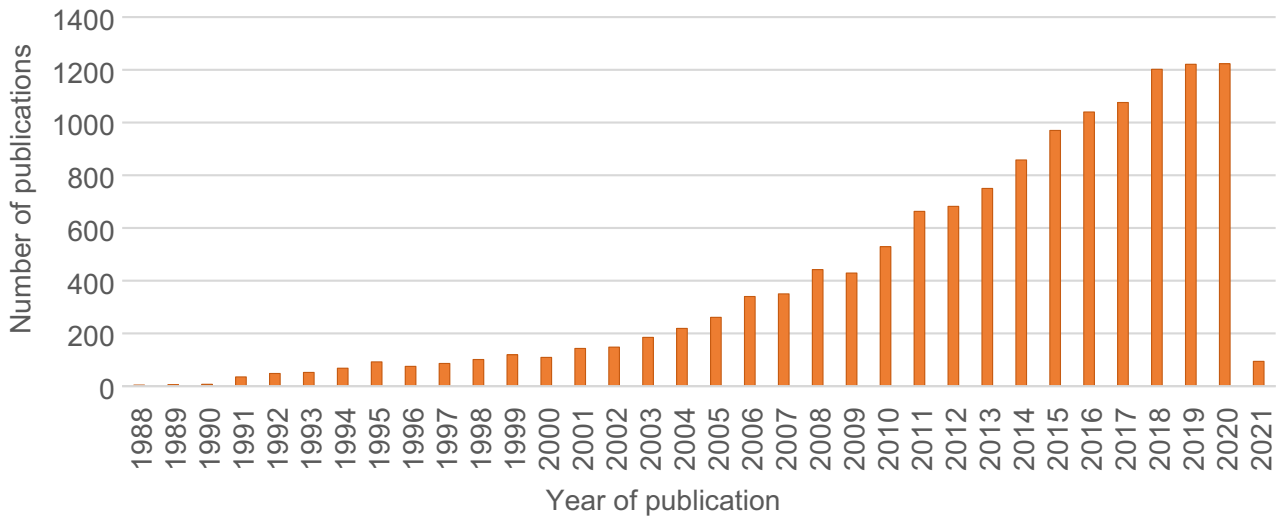

\section{Declarations}

Conflict of interest There are no conflicts of interest to declare.

Publisher's note Springer Nature remains neutral with regard to jurisdictional claims in published maps and institutional affiliations.

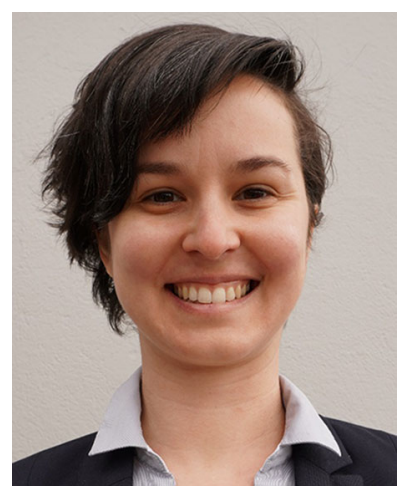

Tiffany Porta Siegel is Assistant Professor at the Department of Imaging Mass Spectrometry, Maastricht MultiModal Molecular Imaging Institute, University of Maastricht, in the Netherlands (2016-2021). Her research group focuses on translation between the development of innovative MS-based instrumentation and direct application to clinical research - with a special interest on intraoperative mass spectrometry and clinical diagnostics. She is an advocate of MSI and actively contributes to its dissemination, she serves as Communication Officer on the executive board of the MSI Society (since 2017) and is Vice-Chair of the European Scientific Committee of MSACL, the Association for Mass Spectrometry and Advances to the Clinical Lab (since 2020). She is co-guest-editor of the special issue on "Biomedical Imaging by High Performance Mass Spectrometry" (IJMS, 2019) and editor of the book "MALDI-MSI: from fundamentals to spatial omics" (RSC, 2021-2022). As of April 2021, she has decided to leave her academic life and focus her expertise in MSI to drug development by joining Boehringer Ingelheim (Biberach, Germany) as Scientist.

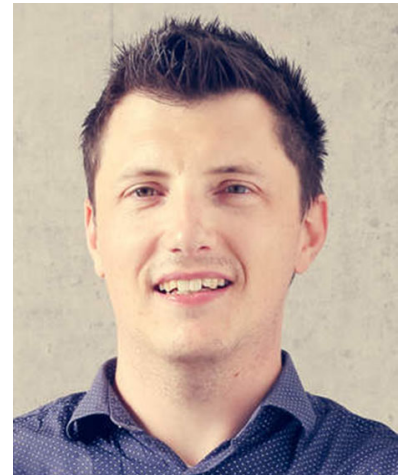

Shane R. Ellis has been an Australian Research Council Future Fellow and Senior Lecturer within Molecular Horizons and the School of Chemistry and Molecular Bioscience at the University of Wollongong, Australia, since 2020. From 2014 to 2019 he was Assistant Professor at the Maastricht MultiModal Molecular Imaging Institute (Maastricht University) where he led the Instrumentation and Application Development group. His research focuses on the continued development and improvement of MSI technology and its applications to challenging biochemical problems. He has a particular interest in lipids and lipidomics and understanding how subtle changes in lipid composition and structure can drive significant biochemical changes and how these are associated with region-specific metabolic changes induced by disease. To this end, he develops new MSI methods allowing for the imaging and identification of lipids at increasing levels of spatial and molecular resolution. He is cochair of the International Mass Spectrometry Foundation (IMSF) "Instrumentation" Focus Group and steering committee member of the "Instrumental and Methodological Developments" interest group within the International Lipidomics Society. 